\title{
Choroidal Neovascular Membrane Association with Tumoral Calcinosis, a Case Report
}

\author{
Karim M Talaat ${ }^{1 *}$, Khalid H Allam ${ }^{1}$, Lujain T Idrees ${ }^{1}$ and Zahid Altaf ${ }^{2}$ \\ ${ }^{1}$ Department of Ophthalmology, National Guard Hospital, Saudi Arabia \\ ${ }^{2}$ Department of Radiology, National Guard Hospital, Saudi Arabia
}

*Corresponding author: Karim Mohamed Talaat, Senior Consultant, Departments of Ophthalmology, Section Head, National Guard Hospital, PO Box 9515, Postal Code 21423, Jeddah, Saudi Arabia

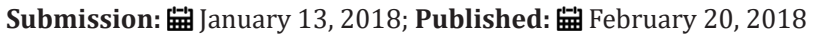

\begin{abstract}
To report a case of Choroidal neovascular membrane in a tumoral calcinosis patient, 2 eyes from 1 patient with choroidal neovascular membrane in one eye, a 40-year-old male with a history of Tumoral calcinosis presented with a choroidal neovascular membrane in his right eye, with his medical work up consisting of elevated levels of phosphorus and normal levels of calcium, with radiological imaging consistent with the diagnosis of tumoral calcinosis. We describe a case of choroidal neovascular membrane in tumoral calcinosis, and its response to Intravitreal anti-VEGF injections.
\end{abstract}

Keywords: Choroidal Neovascular Membrane; Intravitreal Injections; Tumoral Calcinosis

\section{Case Report}

A 40-year-old male with a history of Tumoral calcinosis presented in our clinic on November 2011 complaining of decreased visual acuity in his right eye for the past 2 weeks. His past Medical history included arthritis in his large joints primarily his knees, iron deficiency anemia and hyperphosphatemia. He was on multiple medications including: Cholecalciferol, Ferrous Sulphate, Folic Acid, Omeprazole and Multivitamins. His past Surgical history was unremarkable.

On presentation to our ophthalmic service his visual acuity was 6/21 OD and 6/6 OS. Intraocular pressures were $13 \mathrm{mmhg}$ OD and 12 mmhg OS by Tono-pen measurements. Slit Lamp examination revealed perilimbal Calcification OU nasally otherwise a normal anterior segment examination bilaterally. Fundus examination showed myelinated nerve fibers, punched out chorioretinal lesions in the periphery, angioid streaks and subretinal haemorrhage at the macula with a central retinal thickness of 329 microns with subretinal fluids. Flourescein angiogram confirmed presence of subfoveal choroidal neovascular membrane OD.

Patient received Ranibizumab ( Lucentis, Novartis Pharm. Lim, Horsham, West Sussex, UK) intravitreal injection $6^{\text {th }}$ of December 2011,18 days post intravitreal injection, BCVA right eye 6/24 and OCT shows less subretinal fluid compared to first visit, in December 26 the thickness was 290 microns. Patient had his second lucentis injection on $3^{\text {rd }}$ January 2012. On $24^{\text {th }}$ January 2012 (3 weeks post injection) BCVA right eye 6/21, and OCT showed even less subretinal fluid than what was previously noted.
Third Lucentis Injection was given on $31^{\text {st }}$ of January 2012, 3 weeks later the patient was followed up with a BCVA right eye 6/24 with almost no sub retinal fluid. On $20^{\text {th }}$ of March 2012 BCVA right eye $6 / 60$ with no sub retinal fluid. On $17^{\text {th }}$ of April 2012 BCVA of the right eye 6/36 with a central retinal thickness of 276 microns with no sub retinal fluid.

On 16th of May 2012 BCVA right eye is 6/36 OCT shows a small pocket of sub retinal fluid in which we decide to inject Lucentis 4 th time which was performed on $29^{\text {th }}$ of May 2012 to regain some of the lost visual acuity. Patient was then seen on 11th of June and the BCVA 6/36 and the sub retinal fluid resolved. On $25^{\text {th }}$ of July OCT showed Mild SRF under the fovea. Patient disappeared and showed up $23^{\text {rd }}$ October 2012, with BCVA right eye 6/18-2, and OCT right eye showing a dry macula. On 28 January 2013 BCVA 6/18 OCT Dry Macula. On $13^{\text {th }}$ of March 2013, BCVA 6/60, OCT Macula dry, we thought decrease visual acuity could be due to RPE atrophy, trial of Lucentis advised, which was performed $19^{\text {th }}$ of March 2013.

One-month post injection VA didn't improve and OCT still dry. 3 months later on $24^{\text {th }}$ of July 2013 , BCVA $6 / 30$ and left eye $6 / 6$ patient was complaining of Visual Field disturbance in his left eye Oct left eye shows no sub retinal fluid dry macula which was confirmed with FFA showing NO CNV in left eye. On his last visit on $11 / 8 / 2015$ his vision was CF right eye with no sub retinal fluid in OCT and 6/6 left eye with no changes in OCT.

\section{Discussion}

Tumoral calcinosis, is a subtype of a group of disorders classified under calcinosis cutis. Calcinosis cutis is classified into four types 
being metastatic, Dystrophic, Intraepidermal calcified nodules and Idiopathic of which Tumoral calcinosis is a special form of the Idiopathic type [1], it affects a certain age group of adolescents to young adults [2] with special association and expressibility in afro-Caribbean descent in particular [3]. The Term Tumoral calcinosis was first described in literature in 1943 [4] with certain characteristics as such of elevation of serum phosphate levels, elevated renal tubular phosphate reabsorption and / or normal or elevated serum 1,25-dihydroxyvitamin D [5]. It is characterized with considerable subcutaneous deposits of calcium phosphate near joints of upper and lower limbs such as elbows, hands wrists, hips, knees and feet with these deposits consisting mainly of pleomorphic calcium phosphate crystals. Keeping in mind that calcium and parathyroid hormone levels being on a normal level basis.

It has been linked with certain ophthalmic presentations that are associated with calcification such as calcific lid lesions of the eyelid, Band keratopathy, Conjunctival calcific lesions, angioid streaks and choroidal neovascular membranes [6-14]. To our knowledge this is the Third published case in the English literature that describes the involvement of Tumoral Calcinosis with choriodal neovascular membrane in the retina.

Perimbal calcification was present but no eyelid calcifications which have been mentioned in previous studies [12,13], Radiologic imagining revealed multi lobulated amorphous, cloud-like calcification located in a periarticular distribution adjacent to the right knee, inferior to the right shoulder and around the left hip joint. No significant secondary bone changes.

Recent report in treatment of CNV secondary to angioid streaks in cases of tumoral calcinosis shows great improvement post anti-VEGF injection to affected eyes with CNV after a trial of 3 Lucentis injections and follow up over 6 months [12], however in our particular patient the visual acuity fluctuated in response to Anti-VEGF injections. Follow up period in total was 46 months, which was longer than the previous 2 studies that mentioned the association of CNVM in tumoral calcinosis patients.

With the small number of reports found in relation of tumoral calcinosis associated with $\mathrm{CNV}$ it is difficult to establish a clear

Creative Commons Attribution 4.0

International License

For possible submissions Click Here treatment algorithm, further studies and investigations are needed and patients with tumoral calcinosis are advised to visit the ophthalmic clinic if they complain of changes in vision in order to gain proper management.

\section{Acknowledgments}

We would like to thank Richard Florentino for his aid.

\section{References}

1. Maize J, Metcalf (1997) metabolic diseases of the skin. In: L Elder D (Ed.), Lever's Histopathology of the skin. ( $8^{\text {th }}$ edn), Lippincott- Raven, Philadelphia, USA, pp. 379-382.

2. Datta c, Badnyopadhyay D, Bhattacharyya S, Ghosh S (2005) Tumoral calcinosis. Indian J dermatol Venereol Leprol 71(4): 293-294.

3. Kluger G, Kochs A, Holthausen H (2000) Heterotopic ossification in childhood and adolescence. J Child Neurol 15(6): 406-413.

4. Inclan A, Leon P, Carmejo MG (1943) Tumoral calcinosis. J Am Med Assoc 121(7): 490-495.

5. Mitnick PD, Goldfarb S, Slatopolsky E, Lemann J, Gray RW, et al. (1980) Calcium and phosphate metabolism in tumoral calcinosis. Ann Intern Med 92(4): 482-487.

6. Martinez S, Vogler JB, Harrelson JM (1990) Imaging of tumoral calcinosis: new observations. Radiology 174(1): 215-222.

7. McKee PH, Liomba NG, Hutt MS (1982) Tumoral calcinosis: a pathological study of fifty-six cases. Br J Dermatol 107(6): 669-674.

8. McPhaulJJ,Engel FL(1961)Heterotopiccalcification, hyperphosphatemia and angioid streaks of the retina. Am J Med 31: 488-492.

9. Bruns DE, Lieb W, Conway BP (1988) Band keratopathy and calcific lid lesions in tumoral calcinosis. Case reports. Arch Ophthalmol 106(6): 725-726.

10. Barton DL, Reeves RJ (1961) Tumoral calcinosis. Report of three cases and review of the literature. Am J Roentgenol Radium Ther Nucl Med 86: 351-358.

11. Lafferty FW, Reynolds ES, Pearson OH (1965) Tumoral calcinosis: a metabolic disease of obscure etiology. Am J Med 38: 105-118.

12. Clarke E, Swischuk LE, Hayden CK (1984) Tumoral calcinosis, diaphysitis, and hyperphosphatemia. Radiology 151(3): 643-646.

13. McGrath E, Harney F, Kinsella F (2010) An ocular presentation of familial tumoral calcinosis. Case Rep, BMJ, UK.

14. Bhattacharjee $H$, Bhattacharjee $K$, Yambem $D$ (2014) Ocular involvement in tumoral calcinosis. Indian J Ophthalmol 62(8): 884-887. 